Acta Crystallographica Section E

Structure Reports

Online

ISSN 1600-5368

\section{1-Ethyl-1H-2,1-benzothiazin-4(3H)-one 2,2-dioxide}

\section{Muhammad Shafiq, ${ }^{a}$ Islam Ullah Khan, ${ }^{a}$ M. Nawaz Tahir ${ }^{b}$ * and Waseeq Ahmad Siddiquic}

${ }^{a}$ Government College University, Department of Chemistry, Lahore, Pakistan,

${ }^{\mathbf{b}}$ Department of Physics, University of Sargodha, Sargodha, Pakistan, and

${ }^{\mathbf{c}}$ Department of Chemistry, University of Sargodha, Sargodha, Pakistan

Correspondence e-mail: dmntahir_uos@yahoo.com

Received 14 January 2008; accepted 31 January 2008

Key indicators: single-crystal X-ray study; $T=296 \mathrm{~K}$; mean $\sigma(\mathrm{C}-\mathrm{C})=0.003 \AA$; $R$ factor $=0.043 ; w R$ factor $=0.108 ;$ data-to-parameter ratio $=19.2$.

In the title compound, $\mathrm{C}_{10} \mathrm{H}_{11} \mathrm{NO}_{3} \mathrm{~S}$, there is distorted tetrahedral geometry around the $\mathrm{S}$ atom. The heterocyclic thiazine ring adopts a half-chair conformation. The ethyl and sulfonyl groups form dihedral angles of 82.53 (13) and $88.91(9)^{\circ}$, respectively, with the plane formed by the benzothiazine ring, excluding the $\mathrm{S}$ atom; the $\mathrm{S}$ atom and the ethyl group lie on opposite sides of the ring. The molecules are linked into dimers by intermolecular $\mathrm{C}-\mathrm{H} \cdots \mathrm{O}$ hydrogen bonds involving benzene $\mathrm{C}-\mathrm{H}$ and carbonyl $\mathrm{O}$ atoms, thus forming eight-membered rings. The dimers are linked into chains via interactions of a similar type. There is an intramolecular $\mathrm{C}-\mathrm{H} \cdots \mathrm{O}$ hydrogen bond.

\section{Related literature}

For related literature, see: Hanson et al. (1999); Misu \& Togo (2003); Shafiq et al. (2008); Siddique et al. (2006); Siddiqui et al. (2007); Tahir et al. (2008); Cremer \& Pople (1975).

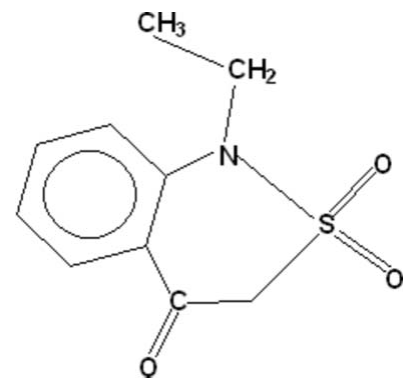

\section{Experimental}

Crystal data

$\mathrm{C}_{10} \mathrm{H}_{11} \mathrm{NO}_{3} \mathrm{~S}$

$M_{r}=225.26$

Triclinic, $P \overline{1}$ $\alpha=99.124(3)^{\circ}$
$\beta=95.075(3)^{\circ}$
$\gamma=104.092(3)^{\circ}$
$V=514.48(4) \AA^{3}$
$Z=2$

Mo $K \alpha$ radiation $\mu=0.30 \mathrm{~mm}^{-1}$

$T=296$ (2) K

$0.15 \times 0.12 \times 0.10 \mathrm{~mm}$

\section{Data collection}

Bruker Kappa APEXII CCD diffractometer

Absorption correction: multi-scan (SADABS; Bruker, 2005)

$T_{\min }=0.965, T_{\max }=0.988$

Refinement

$R\left[F^{2}>2 \sigma\left(F^{2}\right)\right]=0.043$

$w R\left(F^{2}\right)=0.107$

$S=1.02$

2608 reflections

11339 measured reflections 2608 independent reflections 1760 reflections with $I>2 \sigma(I)$ $R_{\text {int }}=0.036$

Table 1

Hydrogen-bond geometry $\left(\AA{ }^{\circ}\right)$.

\begin{tabular}{lllll}
\hline$D-\mathrm{H} \cdots A$ & $D-\mathrm{H}$ & $\mathrm{H} \cdots A$ & $D \cdots A$ & $D-\mathrm{H} \cdots A$ \\
\hline $\mathrm{C} 2-\mathrm{H} 2 \cdots \mathrm{O}^{\mathrm{i}}$ & 0.93 & 2.56 & $3.478(3)$ & 169 \\
$\mathrm{C} 8-\mathrm{H} 8 A \cdots \mathrm{O} 1^{\mathrm{ii}}$ & 0.97 & 2.50 & $3.388(3)$ & 152 \\
$\mathrm{C} 9-\mathrm{H} 9 B \cdots \mathrm{O} 2$ & 0.97 & 2.36 & $2.855(3)$ & 111
\end{tabular}

Symmetry codes: (i) $x, y+1, z$; (ii) $-x+1,-y,-z+2$.

Data collection: $A P E X 2$ (Bruker, 2007); cell refinement: APEX2; data reduction: $S A I N T$ (Bruker, 2007); program(s) used to solve structure: SHELXS97 (Sheldrick, 2008); program(s) used to refine structure: SHELXL97 (Sheldrick, 2008); molecular graphics: ORTEP-3 for Windows (Farrugia, 1997) and PLATON (Spek, 2003); software used to prepare material for publication: Win $G X$ (Farrugia, 1999) and PLATON.

The authors acknowledge the Higher Education Commission, Islamabad, Pakistan, and Bana International, Karachi, Pakistan, for funding the purchase of the diffractometer and for technical support, respectively.

Supplementary data and figures for this paper are available from the IUCr electronic archives (Reference: PV2067).

\section{References}

Bruker (2005). SADABS. Bruker AXS Inc., Madison, Wisconsin, USA.

Bruker (2007). APEX2 (Version 1.27) and SAINT (Version 7.12a). Bruker AXS Inc., Madison, Wisconsin, USA.

Cremer, D. \& Pople, J. A. (1975). J. Am. Chem. Soc. 97, 1354-1358.

Farrugia, L. J. (1997). J. Appl. Cryst. 30, 565.

Farrugia, L. J. (1999). J. Appl. Cryst. 32, 837-838.

Hanson, P. R., Probst, D. A., Robinson, R. E. \& Yau, M. (1999). Tetrahedron Lett. 40, 4761-4764.

Misu, Y. \& Togo, H. (2003). Org. Biomol. Chem. 1, 1342-1346.

Shafiq, M., Tahir, M. N., Khan, I. U., Siddiqui, W. A. \& Arshad, M. N. (2008). Acta Cryst. E64, o389.

Sheldrick, G. M. (2008). Acta Cryst. A64, 112-122.

Siddique, W. A., Ahmad, S., Khan, I. U. \& Malik, A. (2006). J. Chem. Soc. Pak. 28, 583-589.

Siddiqui, W. A., Ahmad, S., Khan, I. U., Siddiqui, H. L. \& Weaver, G. W. (2007). Synth. Commun. 37, 767-773.

Spek, A. L. (2003). J. Appl. Cryst. 36, 7-13.

Tahir, M. N., Shafiq, M., Khan, I. U., Siddiqui, W. A. \& Arshad, M. N. (2008). Acta Cryst. E64, 0557. 


\section{supporting information}

Acta Cryst. (2008). E64, o558 [doi:10.1107/S1600536808003504]

\section{1-Ethyl-1H-2,1-benzothiazin-4(3H)-one 2,2-dioxide}

\section{Muhammad Shafiq, Islam Ullah Khan, M. Nawaz Tahir and Waseeq Ahmad Siddiqui}

\section{S1. Comment}

Sulfonamides in general, and cyclic sulfonamides (sultams) in particular are important therapeutic compounds (Hanson et al., 1999). Among sultams, 1,2-benzothiazine and 2,1-benzothiazine dioxides (benzosultams) have proven to be biologically active (Misu \& Togo, 2003). Due to the importance of 2,1-benzothiazine derivatives in medicinal chemistry, their synthesis has gained enormous attention. After accomplishing the synthesis of a number of 1,2-benzothiazine 1,1dioxide derivatives (Siddique et al., 2006 and Siddiqui et al., 2007), we have recently started the synthesis of various 2,1benzothiazine 2,2-dioxide derivatives.

The title compound, (I), was synthesized in continuation to our research on derivatives of 2,1-benzothiazine. It is a cyclized product of methyl 2-( $N$-ethylmethanesulfonamido)benzoate (Shafiq et al., 2008). The hetrocyclic ring adopts a half chair confirmation which may be described by the puckering parameters (Cremer \& Pople, 19975): Q = 0.554 (2) $\AA$, $\theta=53.6(2)^{\circ}$ and $\varphi=356.2(3)^{\circ}$. The structure of (I) can be best compared with its 1-methyl analogue (Tahir et al., 2008). In (I), the bond distance N1-C9 [1.477 (2) $\AA]$ is significantly longer than the corresponding distance $[1.452$ (2) $\AA]$ in the 1-methyl analogue. The range of bond angles around $\mathrm{S}$ in the two structures are essentially identical. All the atoms in the benzothiazine ring in (I) are nearly planer except that of $\mathrm{S} 1$ which is displaced by 0.783 (2) $\AA$ from the plane defined by $\mathrm{C} 1-\mathrm{C} 8 / \mathrm{N} 1$, while $\mathrm{C} 9$-atom of $N$-ethyl group is at a distance of -0.226 (3) $\AA$. The $N$-ethyl and sulfonyl groups form dihedral angles of $82.53(13)^{\circ}$ and $88.91(9)^{\circ}$, respectively, with the plane formed by $\mathrm{C} 1-\mathrm{C} 8 / \mathrm{N} 1$ atoms. The dihedral angle between these two groups is $46.66(5)^{\circ}$. In the asymmetric unit there is an intramolecular H-bond between $\mathrm{C} 9$ and $\mathrm{O} 2$ atoms. The molecules are dimerized by forming eight member rings through H-bonding between methylene group of thiazine ring and sulfonyl $\mathrm{O}$-atom $(\mathrm{C} 8-\mathrm{H} 8 \cdots \mathrm{O} 1)$. The structure is further stabilized by interactions involving phenyl C$\mathrm{H}$ and carbonyl $\mathrm{O}$-atoms $(\mathrm{C} 2-\mathrm{H} 2 \cdots \mathrm{O} 3)$ linking dimers into chains. Fig. 2 shows hydrogen bonding interactions; details of H-bonding geometry are given in Table 1.

\section{S2. Experimental}

A suspension of hexane-washed sodium hydride ( $4.6 \mathrm{~g}, 96.0 \mathrm{mmol} ., 50 \%$ in mineral oil) was prepared in dry dimethylformamide $(30 \mathrm{ml})$. To this suspension, a solution of methyl 2-( $N$-ethylmethanesulfonamido)benzoate $(19.02 \mathrm{~g}, 74.0$ $\mathrm{mmol})$ in dry dimethylformamide $(70 \mathrm{ml})$ was added. The reaction mixture was stirred at room temperature $(1.5 \mathrm{~h})$ and was poured in a thin stream into hydrochloric acid $(3 \mathrm{~N}, 200 \mathrm{ml})$. The $\mathrm{pH}$ of the mixture was then adjusted to neutral using $\mathrm{NaHCO}_{3}$. After this it was filtered and the filtrate was evaporated under reduced pressure (11 torr) to obtain the title compound (yield; $15 \mathrm{~g}$, 90\%); m.p. 354-355 K. Colorless crystals of (I) suitable for X-ray diffraction were grown from $\mathrm{MeOH}$ by slow evaporation at room temperature. 


\section{S3. Refinement}

$\mathrm{H}$ atoms were positioned geometrically, with $\mathrm{C}-\mathrm{H}=0.93,0.97$, and $0.96 \AA$ for aromatic, methylene and methyl $\mathrm{H}$, and constrained to ride on their parent atoms, with $U_{\text {iso }}(\mathrm{H})=x U_{\text {eq }}(\mathrm{C})$, where $x=1.5$ for methyl $\mathrm{H}$, and $x=1.2$ for all other $\mathrm{H}$ atoms.

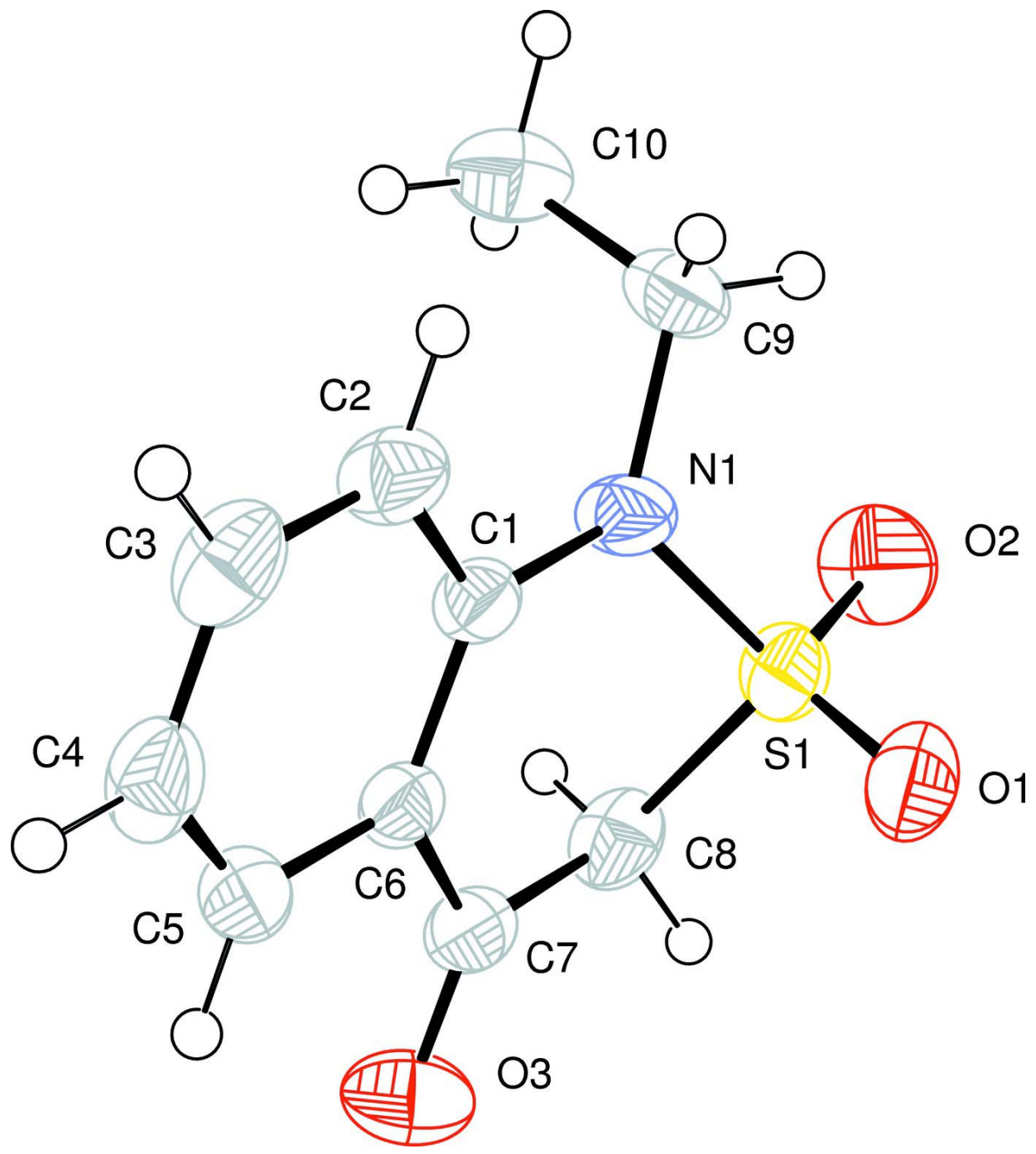

\section{Figure 1}

ORTEP-3 (Farrugia, 1997) drawing of (I) with the atom numbering scheme. The thermal ellipsoids are drawn at the $50 \%$ probability level. 


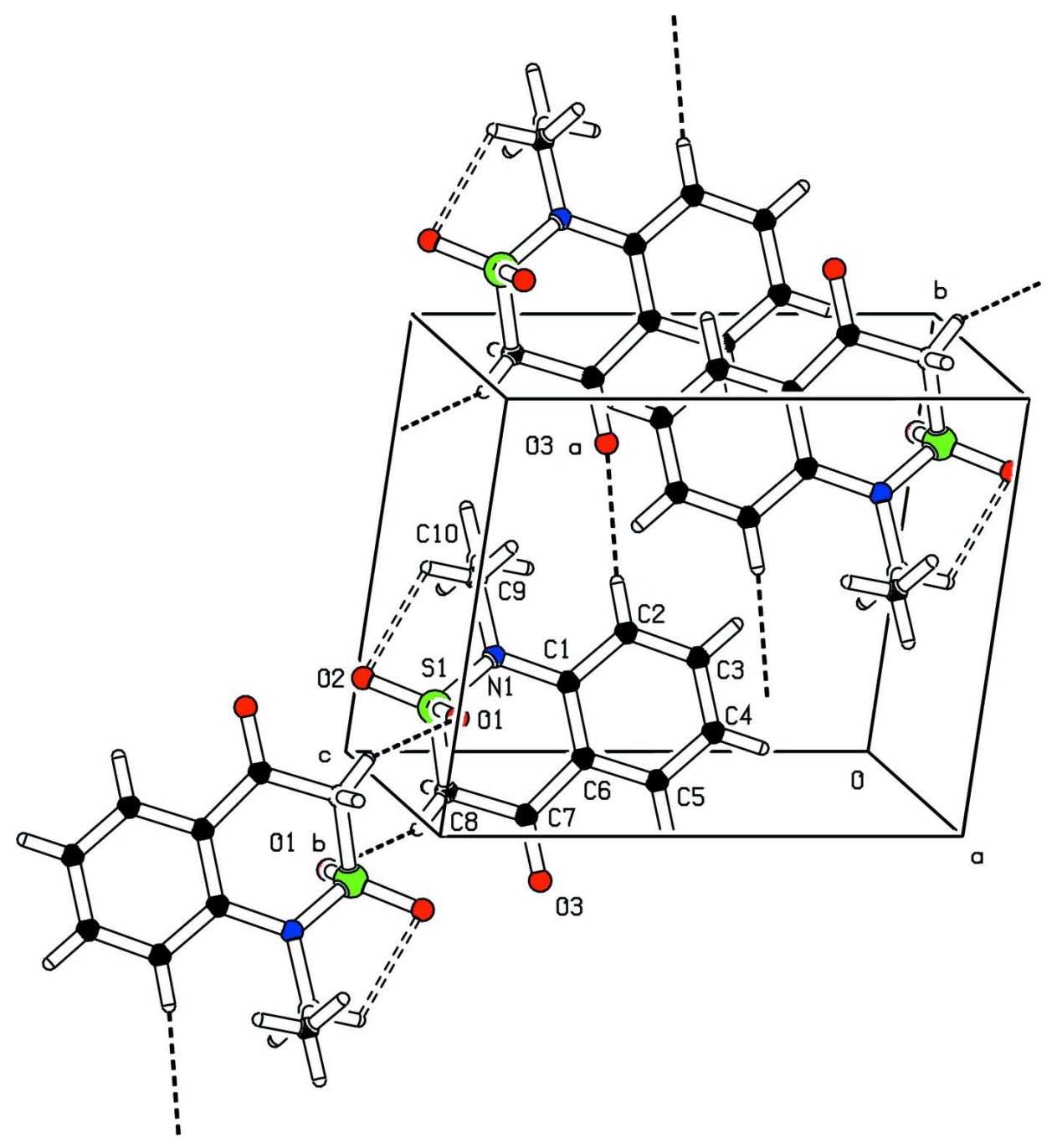

\section{Figure 2}

The unit cell packing of (I) (Spek, 2003) showing the intermolecular hydrogen bonds resulting in dimers.

\section{1-Ethyl-1H-2,1-benzothiazin-4(3H)-one 2,2-dioxide}

\section{Crystal data}

$\mathrm{C}_{10} \mathrm{H}_{11} \mathrm{NO}_{3} \mathrm{~S}$

$M_{r}=225.26$

Triclinic, $P \overline{1}$

Hall symbol: -P 1

$a=7.0272(3) \AA$

$b=8.0448(4) \AA$

$c=9.5880(4) \AA$

$\alpha=99.124(3)^{\circ}$

$\beta=95.075(3)^{\circ}$

$\gamma=104.092(3)^{\circ}$

$V=514.48(4) \AA^{3}$

$Z=2$

$F(000)=236$

$D_{\mathrm{x}}=1.454 \mathrm{Mg} \mathrm{m}^{-3}$

Mo $K \alpha$ radiation, $\lambda=0.71073 \AA$

Cell parameters from 1760 reflections

$\theta=2.2-28.7^{\circ}$

$\mu=0.30 \mathrm{~mm}^{-1}$

$T=296 \mathrm{~K}$

Prismatic, colourless

$0.15 \times 0.12 \times 0.10 \mathrm{~mm}$ 


\section{Data collection}

Bruker Kappa APEXII CCD diffractometer

Radiation source: fine-focus sealed tube Graphite monochromator

Detector resolution: 7.40 pixels $\mathrm{mm}^{-1}$

$\omega$ scans

Absorption correction: multi-scan

(SADABS; Bruker, 2005)

$T_{\text {min }}=0.965, T_{\max }=0.988$

\section{Refinement}

Refinement on $F^{2}$

Least-squares matrix: full

$R\left[F^{2}>2 \sigma\left(F^{2}\right)\right]=0.043$

$w R\left(F^{2}\right)=0.107$

$S=1.02$

2608 reflections

136 parameters

0 restraints

Primary atom site location: structure-invariant direct methods
11339 measured reflections

2608 independent reflections

1760 reflections with $I>2 \sigma(I)$

$R_{\text {int }}=0.036$

$\theta_{\text {max }}=28.7^{\circ}, \theta_{\min }=2.2^{\circ}$

$h=-9 \rightarrow 9$

$k=-10 \rightarrow 10$

$l=-12 \rightarrow 12$
Secondary atom site location: difference Fourier map

Hydrogen site location: inferred from neighbouring sites

$\mathrm{H}$-atom parameters constrained

$w=1 /\left[\sigma^{2}\left(F_{\mathrm{o}}^{2}\right)+(0.0488 P)^{2}+0.0854 P\right]$

where $P=\left(F_{\mathrm{o}}^{2}+2 F_{\mathrm{c}}^{2}\right) / 3$

$(\Delta / \sigma)_{\max }<0.001$

$\Delta \rho_{\max }=0.29$ e $\AA^{-3}$

$\Delta \rho_{\min }=-0.26$ e $\AA^{-3}$

\section{Special details}

Geometry. All e.s.d.'s (except the e.s.d. in the dihedral angle between two 1.s. planes) are estimated using the full covariance matrix. The cell e.s.d.'s are taken into account individually in the estimation of e.s.d.'s in distances, angles and torsion angles; correlations between e.s.d.'s in cell parameters are only used when they are defined by crystal symmetry. An approximate (isotropic) treatment of cell e.s.d.'s is used for estimating e.s.d.'s involving 1.s. planes.

Refinement. Refinement of $F^{2}$ against ALL reflections. The weighted $R$-factor $w R$ and goodness of fit $S$ are based on $F^{2}$, conventional $R$-factors $R$ are based on $F$, with $F$ set to zero for negative $F^{2}$. The threshold expression of $F^{2}>\sigma\left(F^{2}\right)$ is used only for calculating $R$-factors(gt) etc. and is not relevant to the choice of reflections for refinement. $R$-factors based on $F^{2}$ are statistically about twice as large as those based on $F$, and $R$ - factors based on ALL data will be even larger.

Fractional atomic coordinates and isotropic or equivalent isotropic displacement parameters $\left(\AA^{2}\right)$

\begin{tabular}{lllll}
\hline & $x$ & $y$ & $z$ & $U_{\text {iso }} / U_{\text {eq }}$ \\
\hline S1 & $0.32887(8)$ & $0.16467(6)$ & $0.90938(5)$ & $0.04169(17)$ \\
O1 & $0.5349(2)$ & $0.18066(19)$ & $0.90570(14)$ & $0.0531(4)$ \\
O2 & $0.2664(3)$ & $0.2196(2)$ & $1.04236(14)$ & $0.0670(5)$ \\
O3 & $0.2248(2)$ & $-0.25167(19)$ & $0.63616(17)$ & $0.0632(4)$ \\
N1 & $0.2390(2)$ & $0.2638(2)$ & $0.79263(15)$ & $0.0415(4)$ \\
C1 & $0.2558(3)$ & $0.2075(2)$ & $0.64688(17)$ & $0.0328(4)$ \\
C2 & $0.2726(3)$ & $0.3249(3)$ & $0.5533(2)$ & $0.0429(5)$ \\
H2 & 0.2746 & 0.4406 & 0.5861 & $0.051^{*}$ \\
C3 & $0.2864(3)$ & $0.2688(3)$ & $0.4118(2)$ & $0.0489(5)$ \\
H3 & 0.2959 & 0.3475 & 0.3498 & $0.059^{*}$ \\
C4 & $0.2862(3)$ & $0.1000(3)$ & $0.3604(2)$ & $0.0485(5)$ \\
H4 & 0.2982 & 0.0651 & 0.2651 & $0.058^{*}$ \\
C5 & $0.2684(3)$ & $-0.0161(3)$ & $0.45077(19)$ & $0.0420(5)$ \\
H5 & 0.2679 & -0.1310 & 0.4160 & $0.050^{*}$ \\
C6 & $0.2509(2)$ & $0.0337(2)$ & $0.59449(17)$ & $0.0329(4)$ \\
C7 & $0.2276(3)$ & $-0.1022(2)$ & $0.6829(2)$ & $0.0389(4)$
\end{tabular}




\begin{tabular}{lllll} 
C8 & $0.2024(3)$ & $-0.0522(3)$ & $0.8374(2)$ & $0.0445(5)$ \\
H8A & 0.2512 & -0.1285 & 0.8919 & $0.053^{*}$ \\
H8B & 0.0627 & -0.0687 & 0.8454 & $0.053^{*}$ \\
C9 & $0.2066(3)$ & $0.4367(3)$ & $0.8431(2)$ & $0.0461(5)$ \\
H9A & 0.2951 & 0.5239 & 0.8032 & $0.055^{*}$ \\
H9B & 0.2378 & 0.4664 & 0.9461 & $0.055^{*}$ \\
C10 & $-0.0036(3)$ & $0.4394(3)$ & $0.8011(3)$ & $0.0575(6)$ \\
H10A & -0.0197 & 0.5532 & 0.8354 & $0.086^{*}$ \\
H10B & -0.0914 & 0.3545 & 0.8420 & $0.086^{*}$ \\
H10C & -0.0341 & 0.4120 & 0.6992 & $0.086^{*}$ \\
\hline
\end{tabular}

Atomic displacement parameters $\left(\AA^{2}\right)$

\begin{tabular}{lllllll}
\hline & $U^{11}$ & $U^{22}$ & $U^{33}$ & $U^{12}$ & $U^{13}$ & $U^{23}$ \\
\hline S1 & $0.0517(3)$ & $0.0444(3)$ & $0.0303(2)$ & $0.0137(2)$ & $0.00127(19)$ & $0.01149(19)$ \\
O1 & $0.0444(9)$ & $0.0558(9)$ & $0.0541(8)$ & $0.0071(7)$ & $-0.0081(6)$ & $0.0135(7)$ \\
O2 & $0.0971(13)$ & $0.0800(12)$ & $0.0318(7)$ & $0.0339(10)$ & $0.0142(8)$ & $0.0136(7)$ \\
O3 & $0.0782(12)$ & $0.0343(9)$ & $0.0810(11)$ & $0.0192(8)$ & $0.0145(9)$ & $0.0127(7)$ \\
N1 & $0.0633(11)$ & $0.0387(9)$ & $0.0299(7)$ & $0.0260(8)$ & $0.0075(7)$ & $0.0082(6)$ \\
C1 & $0.0347(10)$ & $0.0366(10)$ & $0.0302(8)$ & $0.0141(8)$ & $0.0044(7)$ & $0.0083(7)$ \\
C2 & $0.0504(12)$ & $0.0408(11)$ & $0.0434(10)$ & $0.0167(9)$ & $0.0083(9)$ & $0.0163(8)$ \\
C3 & $0.0492(13)$ & $0.0673(15)$ & $0.0394(10)$ & $0.0197(11)$ & $0.0106(9)$ & $0.0273(10)$ \\
C4 & $0.0436(12)$ & $0.0748(16)$ & $0.0305(9)$ & $0.0224(10)$ & $0.0055(8)$ & $0.0083(9)$ \\
C5 & $0.0374(11)$ & $0.0459(12)$ & $0.0407(10)$ & $0.0152(9)$ & $0.0000(8)$ & $-0.0022(8)$ \\
C6 & $0.0297(9)$ & $0.0366(11)$ & $0.0337(9)$ & $0.0118(8)$ & $0.0026(7)$ & $0.0066(7)$ \\
C7 & $0.0332(10)$ & $0.0337(11)$ & $0.0498(11)$ & $0.0092(8)$ & $0.0013(8)$ & $0.0095(8)$ \\
C8 & $0.0446(12)$ & $0.0431(12)$ & $0.0485(11)$ & $0.0080(9)$ & $0.0024(9)$ & $0.0237(9)$ \\
C9 & $0.0618(14)$ & $0.0334(11)$ & $0.0423(10)$ & $0.0152(10)$ & $0.0087(9)$ & $-0.0004(8)$ \\
C10 & $0.0615(15)$ & $0.0474(14)$ & $0.0693(14)$ & $0.0239(11)$ & $0.0180(11)$ & $0.0075(11)$ \\
& & & & & & \\
\hline
\end{tabular}

Geometric parameters $\left(A,{ }^{\circ}\right)$

\begin{tabular}{llll}
\hline $\mathrm{S} 1-\mathrm{O} 2$ & $1.4244(14)$ & $\mathrm{C} 4-\mathrm{H} 4$ & 0.9300 \\
$\mathrm{~S} 1-\mathrm{O} 1$ & $1.4260(14)$ & $\mathrm{C} 5-\mathrm{C} 6$ & $1.398(2)$ \\
$\mathrm{S} 1-\mathrm{N} 1$ & $1.6405(15)$ & $\mathrm{C} 5-\mathrm{H} 5$ & 0.9300 \\
$\mathrm{~S} 1-\mathrm{C} 8$ & $1.750(2)$ & $\mathrm{C} 6-\mathrm{C} 7$ & $1.473(2)$ \\
$\mathrm{O} 3-\mathrm{C} 7$ & $1.210(2)$ & $\mathrm{C} 7-\mathrm{C} 8$ & $1.510(3)$ \\
$\mathrm{N} 1-\mathrm{C} 1$ & $1.424(2)$ & $\mathrm{C} 8-\mathrm{H} 8 \mathrm{~A}$ & 0.9700 \\
$\mathrm{~N} 1-\mathrm{C} 9$ & $1.477(2)$ & $\mathrm{C} 8-\mathrm{H} 8 \mathrm{~B}$ & 0.9700 \\
$\mathrm{C} 1-\mathrm{C} 2$ & $1.395(2)$ & $\mathrm{C} 9-\mathrm{C} 10$ & $1.503(3)$ \\
$\mathrm{C} 1-\mathrm{C} 6$ & $1.400(2)$ & $\mathrm{C} 9-\mathrm{H} 9 \mathrm{~A}$ & 0.9700 \\
$\mathrm{C} 2-\mathrm{C} 3$ & $1.380(3)$ & $\mathrm{C} 9-\mathrm{H} 9 \mathrm{~B}$ & 0.9700 \\
$\mathrm{C} 2-\mathrm{H} 2$ & 0.9300 & $\mathrm{C} 10-\mathrm{H} 10 \mathrm{~A}$ & 0.9600 \\
$\mathrm{C} 3-\mathrm{C} 4$ & $1.368(3)$ & $\mathrm{C} 10-\mathrm{H} 10 \mathrm{~B}$ & 0.9600 \\
$\mathrm{C} 3-\mathrm{H} 3$ & 0.9300 & $\mathrm{C} 10-\mathrm{H} 10 \mathrm{C}$ & 0.9600 \\
$\mathrm{C} 4-\mathrm{C} 5$ & $1.363(3)$ & & $119.07(16)$ \\
& & &
\end{tabular}




\begin{tabular}{|c|c|c|c|}
\hline $\mathrm{O} 2-\mathrm{S} 1-\mathrm{N} 1$ & $107.40(9)$ & $\mathrm{C} 5-\mathrm{C} 6-\mathrm{C} 7$ & $117.38(17)$ \\
\hline $\mathrm{O} 1-\mathrm{S} 1-\mathrm{N} 1$ & $111.71(9)$ & $\mathrm{C} 1-\mathrm{C} 6-\mathrm{C} 7$ & $123.54(16)$ \\
\hline $\mathrm{O} 2-\mathrm{S} 1-\mathrm{C} 8$ & $110.60(10)$ & $\mathrm{O} 3-\mathrm{C} 7-\mathrm{C} 6$ & $122.76(18)$ \\
\hline $\mathrm{O} 1-\mathrm{S} 1-\mathrm{C} 8$ & $107.58(9)$ & $\mathrm{O} 3-\mathrm{C} 7-\mathrm{C} 8$ & $119.04(17)$ \\
\hline $\mathrm{N} 1-\mathrm{S} 1-\mathrm{C} 8$ & $100.05(8)$ & $\mathrm{C} 6-\mathrm{C} 7-\mathrm{C} 8$ & $118.20(16)$ \\
\hline $\mathrm{C} 1-\mathrm{N} 1-\mathrm{C} 9$ & $121.18(14)$ & $\mathrm{C} 7-\mathrm{C} 8-\mathrm{S} 1$ & $112.13(13)$ \\
\hline $\mathrm{C} 1-\mathrm{N} 1-\mathrm{S} 1$ & $117.15(12)$ & $\mathrm{C} 7-\mathrm{C} 8-\mathrm{H} 8 \mathrm{~A}$ & 109.2 \\
\hline $\mathrm{C} 9-\mathrm{N} 1-\mathrm{S} 1$ & $118.59(12)$ & $\mathrm{S} 1-\mathrm{C} 8-\mathrm{H} 8 \mathrm{~A}$ & 109.2 \\
\hline $\mathrm{C} 2-\mathrm{C} 1-\mathrm{C} 6$ & $119.03(16)$ & $\mathrm{C} 7-\mathrm{C} 8-\mathrm{H} 8 \mathrm{~B}$ & 109.2 \\
\hline $\mathrm{C} 2-\mathrm{C} 1-\mathrm{N} 1$ & $120.08(16)$ & $\mathrm{S} 1-\mathrm{C} 8-\mathrm{H} 8 \mathrm{~B}$ & 109.2 \\
\hline $\mathrm{C} 6-\mathrm{C} 1-\mathrm{N} 1$ & $120.87(15)$ & $\mathrm{H} 8 \mathrm{~A}-\mathrm{C} 8-\mathrm{H} 8 \mathrm{~B}$ & 107.9 \\
\hline $\mathrm{C} 3-\mathrm{C} 2-\mathrm{C} 1$ & $119.70(18)$ & $\mathrm{N} 1-\mathrm{C} 9-\mathrm{C} 10$ & $111.54(17)$ \\
\hline $\mathrm{C} 3-\mathrm{C} 2-\mathrm{H} 2$ & 120.2 & $\mathrm{~N} 1-\mathrm{C} 9-\mathrm{H} 9 \mathrm{~A}$ & 109.3 \\
\hline $\mathrm{C} 1-\mathrm{C} 2-\mathrm{H} 2$ & 120.2 & $\mathrm{C} 10-\mathrm{C} 9-\mathrm{H} 9 \mathrm{~A}$ & 109.3 \\
\hline $\mathrm{C} 4-\mathrm{C} 3-\mathrm{C} 2$ & $121.64(18)$ & N1-C9-H9B & 109.3 \\
\hline $\mathrm{C} 4-\mathrm{C} 3-\mathrm{H} 3$ & 119.2 & $\mathrm{C} 10-\mathrm{C} 9-\mathrm{H} 9 \mathrm{~B}$ & 109.3 \\
\hline $\mathrm{C} 2-\mathrm{C} 3-\mathrm{H} 3$ & 119.2 & $\mathrm{H} 9 \mathrm{~A}-\mathrm{C} 9-\mathrm{H} 9 \mathrm{~B}$ & 108.0 \\
\hline $\mathrm{C} 5-\mathrm{C} 4-\mathrm{C} 3$ & $119.13(18)$ & $\mathrm{C} 9-\mathrm{C} 10-\mathrm{H} 10 \mathrm{~A}$ & 109.5 \\
\hline $\mathrm{C} 5-\mathrm{C} 4-\mathrm{H} 4$ & 120.4 & $\mathrm{C} 9-\mathrm{C} 10-\mathrm{H} 10 \mathrm{~B}$ & 109.5 \\
\hline $\mathrm{C} 3-\mathrm{C} 4-\mathrm{H} 4$ & 120.4 & $\mathrm{H} 10 \mathrm{~A}-\mathrm{C} 10-\mathrm{H} 10 \mathrm{~B}$ & 109.5 \\
\hline $\mathrm{C} 4-\mathrm{C} 5-\mathrm{C} 6$ & $121.41(18)$ & $\mathrm{C} 9-\mathrm{C} 10-\mathrm{H} 10 \mathrm{C}$ & 109.5 \\
\hline $\mathrm{C} 4-\mathrm{C} 5-\mathrm{H} 5$ & 119.3 & $\mathrm{H} 10 \mathrm{~A}-\mathrm{C} 10-\mathrm{H} 10 \mathrm{C}$ & 109.5 \\
\hline $\mathrm{C} 6-\mathrm{C} 5-\mathrm{H} 5$ & 119.3 & $\mathrm{H} 10 \mathrm{~B}-\mathrm{C} 10-\mathrm{H} 10 \mathrm{C}$ & 109.5 \\
\hline $\mathrm{O} 2-\mathrm{S} 1-\mathrm{N} 1-\mathrm{C} 1$ & $-170.18(14)$ & $\mathrm{C} 4-\mathrm{C} 5-\mathrm{C} 6-\mathrm{C} 7$ & $178.61(17)$ \\
\hline $\mathrm{O} 1-\mathrm{S} 1-\mathrm{N} 1-\mathrm{C} 1$ & $58.92(16)$ & $\mathrm{C} 2-\mathrm{C} 1-\mathrm{C} 6-\mathrm{C} 5$ & $1.7(3)$ \\
\hline $\mathrm{C} 8-\mathrm{S} 1-\mathrm{N} 1-\mathrm{C} 1$ & $-54.70(16)$ & $\mathrm{N} 1-\mathrm{C} 1-\mathrm{C} 6-\mathrm{C} 5$ & $-179.84(16)$ \\
\hline $\mathrm{O} 2-\mathrm{S} 1-\mathrm{N} 1-\mathrm{C} 9$ & $29.41(18)$ & $\mathrm{C} 2-\mathrm{C} 1-\mathrm{C} 6-\mathrm{C} 7$ & $-178.16(17)$ \\
\hline $\mathrm{O} 1-\mathrm{S} 1-\mathrm{N} 1-\mathrm{C} 9$ & $-101.49(15)$ & $\mathrm{N} 1-\mathrm{C} 1-\mathrm{C} 6-\mathrm{C} 7$ & $0.3(3)$ \\
\hline $\mathrm{C} 8-\mathrm{S} 1-\mathrm{N} 1-\mathrm{C} 9$ & $144.89(15)$ & $\mathrm{C} 5-\mathrm{C} 6-\mathrm{C} 7-\mathrm{O} 3$ & $0.5(3)$ \\
\hline $\mathrm{C} 9-\mathrm{N} 1-\mathrm{C} 1-\mathrm{C} 2$ & $9.9(3)$ & $\mathrm{C} 1-\mathrm{C} 6-\mathrm{C} 7-\mathrm{O} 3$ & $-179.56(17)$ \\
\hline $\mathrm{S} 1-\mathrm{N} 1-\mathrm{C} 1-\mathrm{C} 2$ & $-149.95(15)$ & $\mathrm{C} 5-\mathrm{C} 6-\mathrm{C} 7-\mathrm{C} 8$ & $-178.23(15)$ \\
\hline $\mathrm{C} 9-\mathrm{N} 1-\mathrm{C} 1-\mathrm{C} 6$ & $-168.48(17)$ & $\mathrm{C} 1-\mathrm{C} 6-\mathrm{C} 7-\mathrm{C} 8$ & $1.7(3)$ \\
\hline $\mathrm{S} 1-\mathrm{N} 1-\mathrm{C} 1-\mathrm{C} 6$ & $31.6(2)$ & $\mathrm{O} 3-\mathrm{C} 7-\mathrm{C} 8-\mathrm{S} 1$ & $149.47(16)$ \\
\hline $\mathrm{C} 6-\mathrm{C} 1-\mathrm{C} 2-\mathrm{C} 3$ & $-0.7(3)$ & $\mathrm{C} 6-\mathrm{C} 7-\mathrm{C} 8-\mathrm{S} 1$ & $-31.7(2)$ \\
\hline $\mathrm{N} 1-\mathrm{C} 1-\mathrm{C} 2-\mathrm{C} 3$ & $-179.18(17)$ & $\mathrm{O} 2-\mathrm{S} 1-\mathrm{C} 8-\mathrm{C} 7$ & $166.56(13)$ \\
\hline $\mathrm{C} 1-\mathrm{C} 2-\mathrm{C} 3-\mathrm{C} 4$ & $-0.8(3)$ & $\mathrm{O} 1-\mathrm{S} 1-\mathrm{C} 8-\mathrm{C} 7$ & $-63.23(15)$ \\
\hline $\mathrm{C} 2-\mathrm{C} 3-\mathrm{C} 4-\mathrm{C} 5$ & $1.2(3)$ & $\mathrm{N} 1-\mathrm{S} 1-\mathrm{C} 8-\mathrm{C} 7$ & $53.53(15)$ \\
\hline $\mathrm{C} 3-\mathrm{C} 4-\mathrm{C} 5-\mathrm{C} 6$ & $-0.2(3)$ & $\mathrm{C} 1-\mathrm{N} 1-\mathrm{C} 9-\mathrm{C} 10$ & $75.8(2)$ \\
\hline $\mathrm{C} 4-\mathrm{C} 5-\mathrm{C} 6-\mathrm{C} 1$ & $-1.3(3)$ & $\mathrm{S} 1-\mathrm{N} 1-\mathrm{C} 9-\mathrm{C} 10$ & $-124.64(16)$ \\
\hline
\end{tabular}

Hydrogen-bond geometry $\left(A,{ }^{\circ}\right)$

\begin{tabular}{lllll}
\hline$D-\mathrm{H} \cdots A$ & $D-\mathrm{H}$ & $\mathrm{H} \cdots A$ & $D \cdots A$ & $D-\mathrm{H}^{\cdots} \cdots A$ \\
\hline $\mathrm{C} 2-\mathrm{H} 2 \cdots \mathrm{O}^{\mathrm{i}}$ & 0.93 & 2.56 & $3.478(3)$ & 169
\end{tabular}


supporting information

$\mathrm{C} 8-\mathrm{H} 8 A \cdots \mathrm{O} 1^{\mathrm{ii}}$

0.97

0.97

2.50

$3.388(3)$

152

$\mathrm{C} 9-\mathrm{H} 9 B \cdots \mathrm{O} 2$

2.36

$2.855(3)$

111

Symmetry codes: (i) $x, y+1, z$; (ii) $-x+1,-y,-z+2$. 